\title{
Postoperative analgesia in bariatric surgery: effect of pre-incisional single dose ketamine
}

\author{
Kasputytė G., Karbonskienė A.
}

Clinic of Anaesthesiology, Faculty of Medicine, Medical Academy

Lithuanian University of Health Sciences, Kaunas, Lithuania

Remifentanil infusions provide excellent intraoperative analgesia with rapid emergence in different patient groups. But postoperative analgesia is still a challenge after this type of anaesthesia. Low-dose intravenous ketamine seems promising in reducing postoperative opioid requirements and opioid induced hyperalgesia.

\section{Aim of study}

To evaluate an effect of pre-incisional single injection of low-dose ketamine on postoperative analgesic requirements and patient satisfaction with postoperative analgesia in laparoscopic bariatric surgery.

\section{Materials and methods}

32 consecutive bariatric patients were randomly assigned to receive single pre-incisional injection of ketamine $(0,15 \mathrm{mg} / \mathrm{kg}$ ) ( $\mathrm{K}$ group) or saline (S group) in a double-blind manner. Standardized protocol of anaesthesia was followed. Propofol $2 \mathrm{mg} / \mathrm{kg}$, Fentanyl 1,2-1,5 mcg/kg, Cis-atracurium 0,1 mg/kg were used for induction. Anaesthesia was maintained with Sevoflurane 0,7-0,8 MAC and remifentanil infusion $0,2-0,5 \mathrm{mcg} / \mathrm{mg} / \mathrm{kg}$. Dosage of medications was based on patients' lean body mass. Postoperative analgesia was provided with bupivacaine infiltration of operative ports and standardized doses of acetaminophen (1 g), ketoprofen (100 $\mathrm{mg})$, intravenous morphine (3-5 $\mathrm{mg}$ ) given 30 mins before end of anaesthesia. Then patients received boluses of morphine $(2 \mathrm{mg})$ on request if pain intensity exceeded score of 5 according to NRS (0-10). Patients were monitored in recovery ward for 2,5 hours and pain intensity was recorded every $15 \mathrm{~min}$. After transfer of patients to regular ward pain intensity was recorded every 6 hours. Patients' satisfaction with analgesia was assessed on the second postoperative day using NRS (0 - unsatisfied, 5 -very satisfied).

Statistical analyses were performed using the Statistical Package for Social Sciences (SPSS) software version 21.0 (SPSS Inc., Chicago, Illinois, USA). Continuous variables are presented as median (min-max) or mean $\pm S D$. Categorical variables are summarized as frequencies. Differences in continuous variables between the two groups were evaluated using the independent samples $t$ test or the Mann-Whitney $U$ test. Categorical variables were compared using the Pearson $\chi^{2}$ test. A $P$ value less than 0.05 was considered significant.

\section{Results}

Both groups did not differ in demographic values, BMI, operative time and opioid consumption (table 1 ).

Table 1. Patient characteristics, duration of surgery and anaesthesia and total dose of opioids in each group

\begin{tabular}{|l|c|c|c|}
\hline \multicolumn{1}{|c|}{ Variable } & $\begin{array}{c}\text { Saline group (S) } \\
\text { (control) }\end{array}$ & $\begin{array}{c}\text { Ketamine group } \\
(\mathrm{K})\end{array}$ & $\begin{array}{c}\mathrm{P}- \\
\text { value }\end{array}$ \\
\hline Sex (male/female) & $5 / 12$ & $4 / 11$ & 0,86 \\
\hline Age (yrs )* & $46(28-64)$ & $45(18-66)$ & 0,41 \\
\hline Body mass index* & $45,0(34,6-55,4)$ & $43,9(34,9-55,3)$ & 0,68 \\
\hline Lean body mass $(\mathrm{kg})^{*}$ & $86,7(71,2-103,8)$ & $81,7(74,9-100,5)$ & 0,94 \\
\hline Operative time $(\mathrm{min})^{*}$ & $89(56,0-144,0)$ & $78,0(59,0-115,0)$ & 0,33 \\
\hline Anaesthesia time $(\mathrm{min})^{*}$ & $112(95-165)$ & $112(95-150)$ & 0,53 \\
\hline Intraoperative remifentanyl $(\mathrm{mg})^{*}$ & $1,8(0,75-3,0)$ & $2,0(0,5-3,2)$ & 0,94 \\
\hline Total morphine dose $(\mathrm{mg})^{*}$ & $10,0(7,0-12,5)$ & $9,0(3,0-15,0)$ & 0,022 \\
\hline
\end{tabular}

*Data are presented as median (min-max)

Both groups demonstrated similar pain scores postoperatively $(p>0,05)$ (picture 1$)$.

Picture 1. Postoperative pain scores.

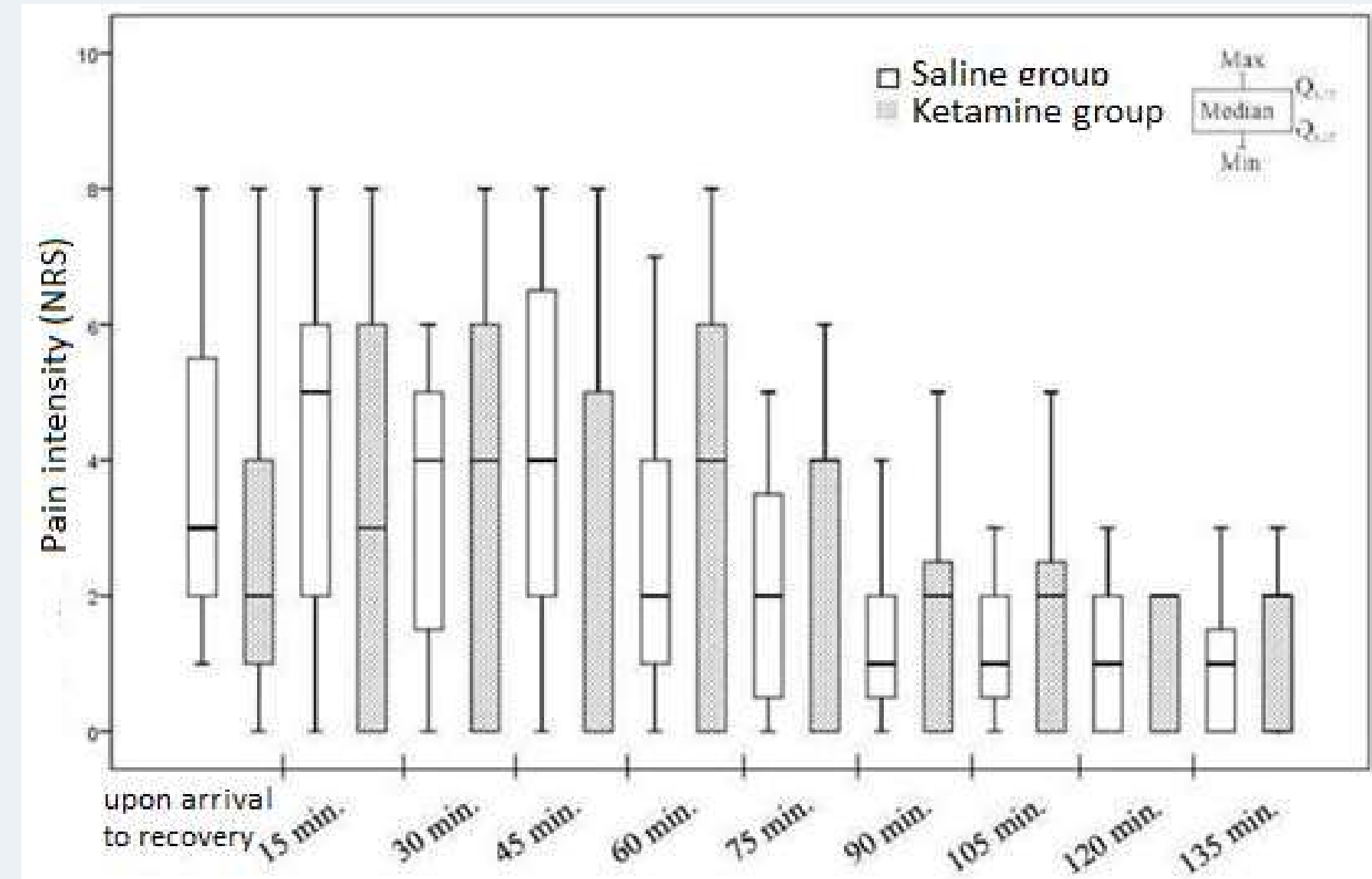

Patients' satisfaction with analgesia was similarly high in both groups $(p>0,05)$ (picture 2$)$.

Picture 2. Patients' satisfaction with analgesia.
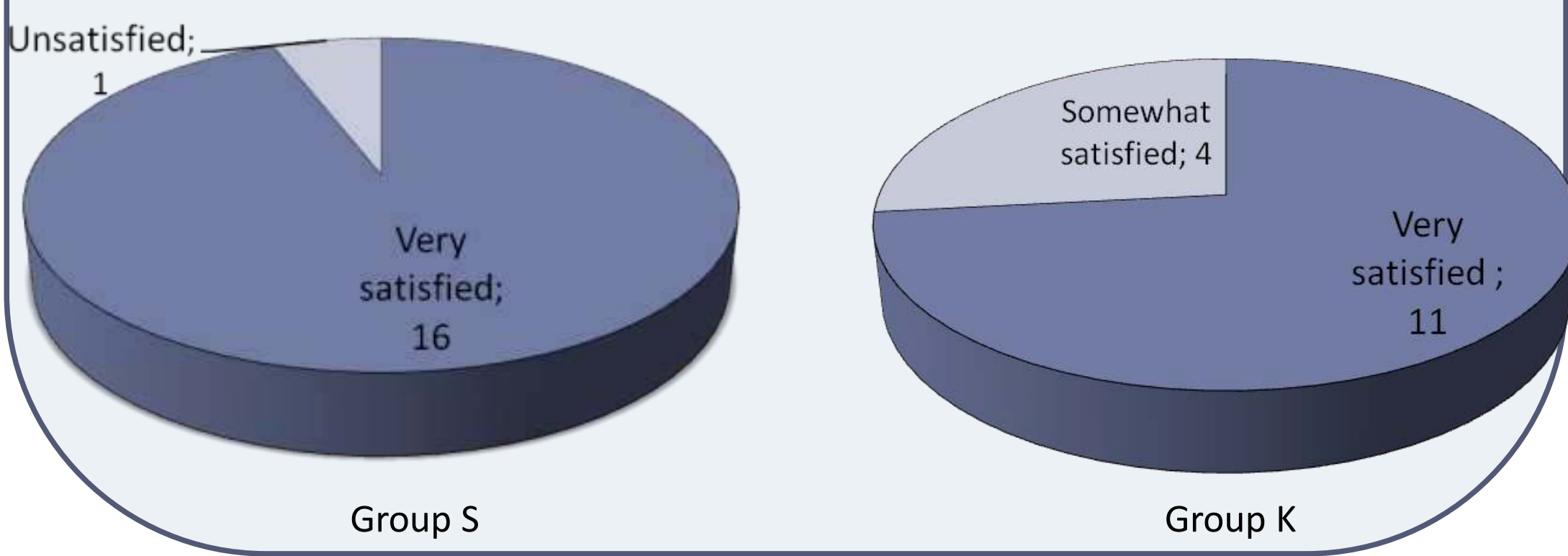

\section{Conclusion}

Pre - incisional single dose ketamine results in slight reduction of morphine consuption postoperatively. High patient satisfaction is achieved if multimodal analgesia protocol is followed. 